\title{
Elimination of Staphylococcus aureus nasal carriage in intensive care patients lowers infection rates
}

\author{
Leila Akhtar Danesh ${ }^{1} \cdot$ Zeinab Saiedi Nejad ${ }^{2} \cdot$ Hossein Sarmadian ${ }^{2} \cdot$ Saeed Fooladvand $^{1} \cdot$ Alex van Belkum $^{3}$. \\ Ehsanollah Ghaznavi-Rad ${ }^{1,4,5}$
}

Received: 20 September 2019 / Accepted: 20 September 2019 / Published online: 12 November 2019

(C) Springer-Verlag GmbH Germany, part of Springer Nature 2019

\begin{abstract}
This study surveys the clinical relevance of the nasal Staphylococcus aureus colonization status on intensive care unit (ICU)acquired S. aureus infections and compares molecular characteristics of isolates from the nose and infectious sites. The 390 patients included comprised 278 non-carriers and 112 carriers. Among the carriers, 56 were decolonized with mupirocin. Decolonization was verified through a second (negative) culture. Spa typing and virulence gene profiling were performed for all isolates. Twenty six $S$. aureus infections were detected in the carriage group and 20 in the non-carriage group. Eighteen of these $26(69.2 \%)$ infections were among carriers, and 8 of these $26(30.8 \%)$ infections occurred among decolonized carriers $(p=$ 0.02). Overall, 31/112 (27.7\%) of the colonized patients and 25/46 (60.1\%) of infection were due to methicillin-resistant $S$. aureus (MRSA). The highest frequency virulence genes were sea and $h l g$ (both 100\%) in nasal isolates and sea, $h l g$, fnb, and $c l f$ $(100 \%)$ for infectious isolates. t030 was the most abundant spa type overall. $S$. aureus carriers were more likely to develop $S$. aureus infection compared with decolonized and non-carrying patients. The sources of ICU S. aureus infection appear to be exogenous mostly, and a predominant clone (spa type 030) plays an important role. We confirm that nasal mupirocin treatment prevents ICU infections even when there is an increased prevalence of nosocomial MRSA.
\end{abstract}

Keywords Staphylococcus aureus $\cdot$ Nasal carriage $\cdot$ Nosocomial infections $\cdot$ Intensive care unit

\section{Introduction}

Staphylococcus aureus is an opportunistic human pathogen and part of the commensal flora. It causes many life-threatening infections in patients in intensive care units (ICUs). This

Leila Akhtar Danesh and Zeinab Saiedi Nejad contributed equally to this study and are co-first authors.

Ehsanollah Ghaznavi-Rad

e.ghaznavirad@arakmu.ac.ir; ghaznaviehs@yahoo.com

1 Department of Medical Microbiology and Immunology, Faculty of Medicine, Arak University of Medical Sciences, Arak, Iran

2 Department of Infectious Diseases, Faculty of Medicine, Arak University of Medical Sciences, Arak, Iran

3 Clinical Unit, BioMérieux, La Balme les Grottes, France

4 Molecular and Medicine Research Center, Arak University of Medical Sciences, Arak, Iran

5 Department of Medical Microbiology, Faculty of Medicine, Arak University of Medical Sciences, Arak, Iran includes bacteremia, ventilator-associated pneumonia, infections related to the presence of indwelling medical devices and wound, and surgical site infections. These infections are a leading cause of prolonged hospital stay, additional antibiotic use, higher morbidity and mortality, and increased healthcareassociated costs (e.g., [1]). Several virulence factors play a role in the pathogenicity of $S$. aureus including toxic shock syndrome toxin 1 (TSST-1), Panton-Valentine leukocidin $(P V L)$, enterotoxins, hemolysin, exfoliative toxins, and various adhesive proteins [2]. Methicillin-resistant S. aureus (MRSA) strains acquired the mecA gene-containing staphylococcal cassette chromosome (SCCmec) [3]. Increased emergence of nosocomial multidrug resistance among MRSA strains has become a global concern in hospital environments [4]. About 30\% of humans are nasal carriers of $S$. aureus and have a greater risk to develop endo-infections [5]. Thus, screening of high-risk patients (MRSA or methicillin-susceptible $S$. aureus (MSSA)-colonized) and eradication of $S$. aureus nasal carriage are essential for reducing the overall frequency of $S$. aureus infection in hospital settings and particularly in ICUs $[5,6]$. In this regard, molecular typing methods are useful in tracing and monitoring 
of nosocomial outbreaks [7]. The purpose of this study was to determine the role of nasal $S$. aureus colonization in ICUacquired $S$. aureus infections.

\section{Materials and methods}

\section{Study design}

The Vali-Asr Hospital is the largest medical training center in the city of Arak, capital of the central province in Iran. The current study was performed during a 9-month period (December 2013 to September 2014), and this trial was registered at the Iranian Registry of Clinical Trials with IRCT number 92-153-6. During this episode, the $S$. aureus nasal colonization status was checked by culture for all patients at admission in ICUs. Patients were classified into two groups: carriers and non-carriers. Half of the carriers were decolonized using mupirocin, confirmed by a negative nasal follow-up culture [8]. Patients were admitted to the ICU and screened for $S$. aureus infection during their stay. Exclusion criteria included allergy to mupirocin, taking antibiotics, a stay in the ICU $<48 \mathrm{~h}$, a known $S$. aureus infection at ICU admission, or the development of an $S$. aureus infection during the first $48 \mathrm{~h}$ of ICU stay. Nasal and ICU-acquired $S$. aureus infectious isolates were investigated by spa typing, for methicillin resistance and for the presence of virulence genes.

\section{S. aureus isolation and identification}

Nasal swabs and infectious site samples were obtained by trained personnel. Sampling and transport were performed using the Transwab system and Amies transport medium (Medical Wire and Equipment Company, Corsham, UK). All samples were transferred to the Laboratory of Microbiology within 30 $\mathrm{min}$. The specimens were incubated at $37^{\circ} \mathrm{C}$ for $6-12 \mathrm{~h}$, and swabs were cultured on sheep blood agar and mannitol salt agar. Plates were aerobically incubated at $37^{\circ} \mathrm{C}$ for $24-48 \mathrm{~h}$, after which smears were Gram-stained and biochemical tests as well as ssa442 PCR were performed for identification of S. aureus suspect colonies. All $S$. aureus isolates were stored in LuriaBertani (LB) broth with $20 \%$ glycerol at $-70{ }^{\circ} \mathrm{C}$.

\section{MecA and virulence genes' PCR}

Purification of DNA was accomplished using the Tissue Genomic DNA Extraction Mini Kit (FavorPrep, FavorGen, Taiwan) following the manufacturer's instructions. All PCRs were performed in a total volume of $25 \mu$ l containing $12.5 \mu \mathrm{l}$ Super PCR Mastermix 2X (Taq DNA polymerase, dNTP, $\left.\mathrm{MgCl}_{2}\right), 1 \mu \mathrm{l}$ of each primer $(10 \mu \mathrm{M}), 1 \mu \mathrm{l}$ template DNA $(100 \mathrm{ng})$, and $9.5 \mu \mathrm{l}$ nuclease-free water. PCR runs were carried out on a peQlab Thermocycler (peQlab, UK). The conditions were as follows: initial denaturation at $94{ }^{\circ} \mathrm{C}$ for 5 min, 30 cycles at $94^{\circ} \mathrm{C}$ for $1 \mathrm{~min}$ with annealing temperature at $72{ }^{\circ} \mathrm{C}$ for $1 \mathrm{~min}$ in between, followed by a final extension at $72{ }^{\circ} \mathrm{C}$ for $5 \mathrm{~min}$. Amplified DNA was visualized using electrophoresis in $1 \%$ agarose gel in $1 \times$ Tris-EDTA buffer.

\section{Molecular spa typing}

Spa typing was performed by PCR aimed at the polymorphic $\mathrm{X}$ region of the protein $\mathrm{A}$ gene and sequencing (Gene Fanavaran Company, Iran). Sequences were edited using the Chromas software (version 2.6.2, Australia). Spa types were assigned following the guidelines of the Ridom Spa database (http://www.spaserver.ridom.de). All S. aureus isolates from prior colonized patients $(n=56)$, decolonized patients $(n=56)$ , and infectious sites $(n=46)$ were spa-typed.

\section{Statistical analysis}

The frequency and proportions of $m e c A$, virulence genes, and spa types between groups of isolates were calculated by SPSS version 24 (Microsoft Office, Chicago, IL, USA, ver. 24) by applying the Pearson chi-square test with significance at $p \leq 0.05$.

\section{Results}

\section{Carriage status and infection isolates}

In total, 490 patients were enrolled and 100 patients were excluded for reasons mentioned above. Among the remaining 390 participants, $112(28.7 \%)$ were nasal S. aureus carriers and $278(71.3 \%)$ were not colonized. Colonized patients were segregated into prior colonized and decolonized groups (each 56 cases). During the study period, $46(11.8 \%)$ cases were infected with $S$. aureus. Twenty-six (56.5\%) were carriers, 18 (69.2\%( were prior colonized, and $8(30.7 \%)$ were among the decolonized. Twenty (43.5\%) were in the non-colonized group. The number of $S$. aureus infections in prior carriers was significantly higher than in decolonized patients $(p<0.05)$. Table 1 provides additional clinical detail.

\section{Methicillin resistance and virulence genes' profiles}

Out of the 112 S. aureus strains, 31 cases $(27.7 \%)$ were MRSA and $81(72.3 \%)$ were MSSA. Among 46 infectious isolates, 25 $(54.3 \%)$ were MRSA and 21 (45.7\%) were MSSA. A significantly higher MRSA frequency was found among patients with infections compared with nasal isolates only $(p<0.05)$. In $S$. aureus isolated from nasal cavities, the highest frequency virulence factor genes were sea and $h l g$ (both 100\%) followed by fnb (98.2\%), clf (97.3\%), can (64.3\%), arcA (63.4\%), pvl (8\%), and sec (1.8\%) (Table 2). In S. aureus isolated from infections, 
Table 1 Frequency of $S$. aureus isolated from sites of infection

\begin{tabular}{|c|c|c|c|c|c|c|c|}
\hline \multirow{2}{*}{\multicolumn{2}{|c|}{ S. aureus isolated from: }} & \multicolumn{6}{|c|}{ Infectious sites } \\
\hline & & Lung & Wound & Blood & Urine & $\mathrm{CSF}$ & Total \\
\hline \multicolumn{2}{|c|}{ Non-carrier } & 10 & 6 & 3 & 1 & 0 & $20(43.5 \%)$ \\
\hline \multirow[t]{2}{*}{ Carrier } & Prior colonization & 13 & 3 & 1 & 0 & 1 & $18(39.1 \%)$ \\
\hline & Decolonized & 5 & 2 & 0 & 0 & 1 & $8(17.4 \%)$ \\
\hline \multicolumn{2}{|l|}{ Total } & $28(60.8 \%)$ & $11(23.9 \%)$ & $4(8.7 \%)$ & $1(2.2 \%)$ & $2(4.3 \%)$ & $46(100 \%)$ \\
\hline
\end{tabular}

the most frequent virulence factor genes were sea, $h l g, f n b$, and clf (all at $100 \%$ ) followed by can (93.5\%), arcA (71.7\%), and $p v l$ (17.4\%), whereas sec was not found (Table 2). There was a significantly higher $\operatorname{arc} A$ frequency in stains from infections $(p$ $<0.05$ ). The frequencies of sea, sec, $h l g, f n b, c l f$, can, and $p v l$ genes did not differ $(p>0.05)$.

\section{Spa typing}

Spa typing defined 19 different types (Table 3 ). The most frequent spa type was t030: $43(38.4 \%)$ for carriers and 28 $(60.9 \%)$ for infectious isolates. The spa type $\mathrm{t} 14386$ was first reported in Iran. Spa types t019, t346, t376, t969, t1149, $\mathrm{t} 3649$, and $\mathrm{t} 4242$ were detected only in isolates from infectious sites. Spa types t238, t304, t136, t491, t1358, t14386, and 44242 were detected only in isolates from nasal cavities. Spa types t030, t084, t012, t021, t325, and 1937 were detected in both groups (Fig. 1). Thirteen identical spa types were found for infectious and colonizing isolates from individual patients suggesting autoinfection.

\section{Discussion}

\section{Carriage and infection}

Our patients displayed a similar nasal carriage rate for $S$. aureus $(28.7 \%)$ as previously reported for the population of central Iran (22.1\% [9] and 20.1\% [10]). The MRSA nasal colonization rate $(27.7 \%)$ was higher than reported for institutes in the USA [11], China [12], and Turkey [13], though lower than the 46\% recorded in Brazil [14]. A high MRSA prevalence of $54.3 \%$ was found in this study which was comparable with that found by previous local studies $(42.8 \%$ and $42 \%)[15,16]$. The frequency of MRSA in infection was two times $(54.3 \%$ versus $27.67 \%$ ) higher than in nasal specimens $(p=0.01)$ which indicated the presence of endemic MRSA in our ICUs. This likely causes an increased risk of MRSA acquisition. Our $S$. aureus infection incidence in ICUs (11.8\%) was much higher than the $2 \%, 2.3 \%$, and $6 \%$ recorded in previous studies $[17,18]$. S. aureus-colonized patients had a 1.3 times increased risk of ICU-acquired S. aureus infection compared with non-carriers (56.5\% versus $43.5 \%)$.

\section{Virulence gene profiles}

The $p v l$ positivity rate among clinical isolates (17.4\%) was higher than among nasal isolates (8\%). Ayepola et al. [19] showed that the $p v l$ prevalence among infectious isolates $(80.2 \%)$ was higher than among isolates from carriers

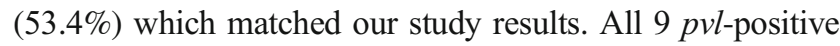
S. aureus isolates obtained from nasal cavities were MSSA, and a significant difference was found between $p v l$-positive strains and the absence of the mecA gene $(p=0.01)$. The prevalence of the $p v l$ gene in our MSSA isolates was higher than that in MRSA isolates and in another study from our

Table 2 Virulence gene frequencies of $S$. aureus isolated from infectious sites and the nasal cavity of colonized patients

\begin{tabular}{|c|c|c|c|c|c|c|c|c|c|c|}
\hline \multicolumn{2}{|c|}{ S. aureus isolated from: } & \multicolumn{8}{|c|}{ Virulence genes } & \multirow[t]{2}{*}{ mecA } \\
\hline & & Sea & $h l g$ & $f n b$ & clf & cna & $\operatorname{arcA}$ & $P v l$ & $\sec$ & \\
\hline \multirow[t]{4}{*}{ Infectious sites } & $\begin{array}{l}\text { Non-colonized } \\
\quad(n=20)\end{array}$ & 20 & 20 & 20 & 20 & 19 & 15 & 7 & 0 & 8 \\
\hline & $\begin{array}{l}\text { Prior colonized } \\
\quad(n=18)\end{array}$ & 18 & 18 & 18 & 18 & 17 & 13 & 1 & 0 & 7 \\
\hline & $\begin{array}{l}\text { Decolonized } \\
\quad(n=8)\end{array}$ & 8 & 8 & 8 & 8 & 6 & 5 & 0 & 0 & 10 \\
\hline & Total $(n=46)$ & $46(100 \%)$ & $46(100 \%)$ & $46(100 \%)$ & $46(100 \%)$ & $43(93.5 \%)$ & $33(71.7 \%)$ & $8(17.4 \%)$ & 0 & $25(54.3 \%)$ \\
\hline $\begin{array}{l}\text { Nasal cavity } \\
\quad(n=112)\end{array}$ & & $112(100 \%)$ & $112(100 \%)$ & $110(98.2 \%)$ & $109(97.3 \%)$ & $72(64.3 \%)$ & $71(63.4 \%)$ & $9(8 \%)$ & $2(1.8 \%)$ & $31(27.6 \%)$ \\
\hline
\end{tabular}


Table 3 Spa types of infectious and colonizing $S$. aureus isolates

\begin{tabular}{|c|c|c|c|c|c|c|}
\hline \multirow[t]{2}{*}{ Number } & \multirow{2}{*}{$\begin{array}{l}\text { Spa } \\
\text { type }\end{array}$} & \multicolumn{4}{|c|}{ Infection isolates } & \multirow{2}{*}{$\begin{array}{l}\text { Colonization } \\
\text { isolates }\end{array}$} \\
\hline & & $\begin{array}{l}\text { Non- } \\
\text { colonized }\end{array}$ & $\begin{array}{l}\text { Prior } \\
\text { colonized }\end{array}$ & Decolonized & Total & \\
\hline 1 & t030 & 12 & 10 & 6 & $\begin{array}{l}28 \\
\quad(60.9 \%)\end{array}$ & $43(38.4 \%)$ \\
\hline 2 & t084 & 5 & 0 & 0 & $5(10.8 \%)$ & $5(4.5 \%)$ \\
\hline 3 & t012 & 1 & 2 & 0 & $3(6.5 \%)$ & $15(13.4 \%)$ \\
\hline 4 & t021 & 0 & 1 & 0 & $1(2.17 \%)$ & $37(33 \%)$ \\
\hline 5 & t019 & 0 & 1 & 0 & $1(2.17 \%)$ & 0 \\
\hline 6 & $\mathrm{t} 325$ & 0 & 0 & 1 & $1(2.17 \%)$ & $1(0.9 \%)$ \\
\hline 7 & $\mathrm{t} 346$ & 0 & 1 & 0 & $1(2.17 \%)$ & 0 \\
\hline 8 & $\mathrm{t} 376$ & 1 & 0 & 0 & $1(2.17 \%)$ & 0 \\
\hline 9 & t937 & 0 & 0 & 1 & $1(2.17 \%)$ & $2(1.8 \%)$ \\
\hline 10 & t969 & 0 & 1 & 0 & $1(2.17 \%)$ & 0 \\
\hline 11 & t1149 & 1 & 0 & 0 & $1(2.17 \%)$ & 0 \\
\hline 12 & t3649 & 0 & 1 & 0 & $1(2.17 \%)$ & 0 \\
\hline 13 & $\mathrm{t} 4242$ & 0 & 1 & 0 & $1(2.17 \%)$ & 0 \\
\hline 14 & $\mathrm{t} 238$ & 0 & 0 & 0 & 0 & $2(1.8 \%)$ \\
\hline 15 & $\mathrm{t} 304$ & 0 & 0 & 0 & 0 & $2(1.8 \%)$ \\
\hline 16 & $\mathrm{t} 136$ & 0 & 0 & 0 & 0 & $1(0.9 \%)$ \\
\hline 17 & t491 & 0 & 0 & 0 & 0 & $2(1.8 \%)$ \\
\hline 18 & $\mathrm{t} 1358$ & 0 & 0 & 0 & 0 & $1(0.9 \%)$ \\
\hline 19 & $\mathrm{t} 14386$ & 0 & 0 & 0 & 0 & $1(0.9 \%)$ \\
\hline 20 & Total & 20 & 18 & 8 & $46(100 \%)$ & $112(100 \%)$ \\
\hline
\end{tabular}

region [20]. All clinical and nasal isolates in this latter study carried the sea gene while the sec gene was absent in clinical isolates and found only in $1.8 \%$ of the nasal isolates. Nashev et al. [21] reported sea as the most abundant enterotoxin gene in $S$. aureus isolated from nasal specimens. In contrast, the sec gene was the most abundant enterotoxin encoding gene, followed by sea, sed, and seb, with a lower frequency in a study from Tehran [22]. Normannoa et al. [23] reported the highest levels of SED, followed by SEC, SEA, and SEB. In this study, the frequency of acme in clinical isolates $(96.4 \%)$ was higher than in nasal isolates $(63.4 \%)$. We found that $c l f$ and $f n b$ were present among all clinical isolates and that can was observed among 93.5\%. The same genes were less frequent in nasal isolates $(97.3 \%$ for clf, $98.2 \%$ for $f n b$, and $64.3 \%$ for can). Ghasemian et al. [24] showed that the $c l f$ gene was present among all isolates and recorded 63\%,6\%, and $63 \%$ for $f n b A, f n b B$, and $c n a$, respectively. Similar to our results, Duran et al. [25] showed that $78.4 \%$ of their isolates carried the cna gene.

\section{Spa typing}

Spa typing revealed a broad range of types among both groups of isolates, 13 different spa types for strains from infections and 12 types for isolates from the nasal cavity. In a previous study in Iran, 11 different spa types were identified among $S$. aureus isolated from ICUs. Overall, $43 \%$ of these isolates belonged to spa types t030 and t037 [26]. In another study [27], only five different spa types were found among $S$. aureus isolates from ICUs of general hospitals in Tehran, Iran. The majority of the isolates belonged to spa types $t 970(69.9 \%)$ and again t030 (33.3\%). Also, t084 (74.8\%), t2304 (5\%), and t8441 (4.6\%) were abundant in clinical isolates, and spa types t084 (38/7\%), t091 (10/7\%), t1931 (4\%), and t8435 (4\%) were most frequent among carrier isolates [19].

In our study, the spa type 030 was most common. This result is in concordance with the findings of Chen et al. [28] who showed that t030 (52\%) was the most frequent clone among their Chinese isolates. In another study, the spa type t030 was the second most common [29]. A study from Malaysia showed that spa types t037, t10562, and t0421 were most common among carriers and spa types t037, t4184, and t10562 were the most frequent among clinical isolates [5]. In another study in Iran, spa types $\mathrm{t304}$ and $\mathrm{t} 7688$ were reported as the most abundant types among clinical isolates [30]. ST239 MRSA has been reported as the dominant clone in Iranian hospitals [31], many of which were of the spa type t030, demonstrating the significant local spread of this type. It can be concluded that many infections acquired in the ICU originate from within the hospital and are hence exogenous. 
Fig. 1 Summary of the study

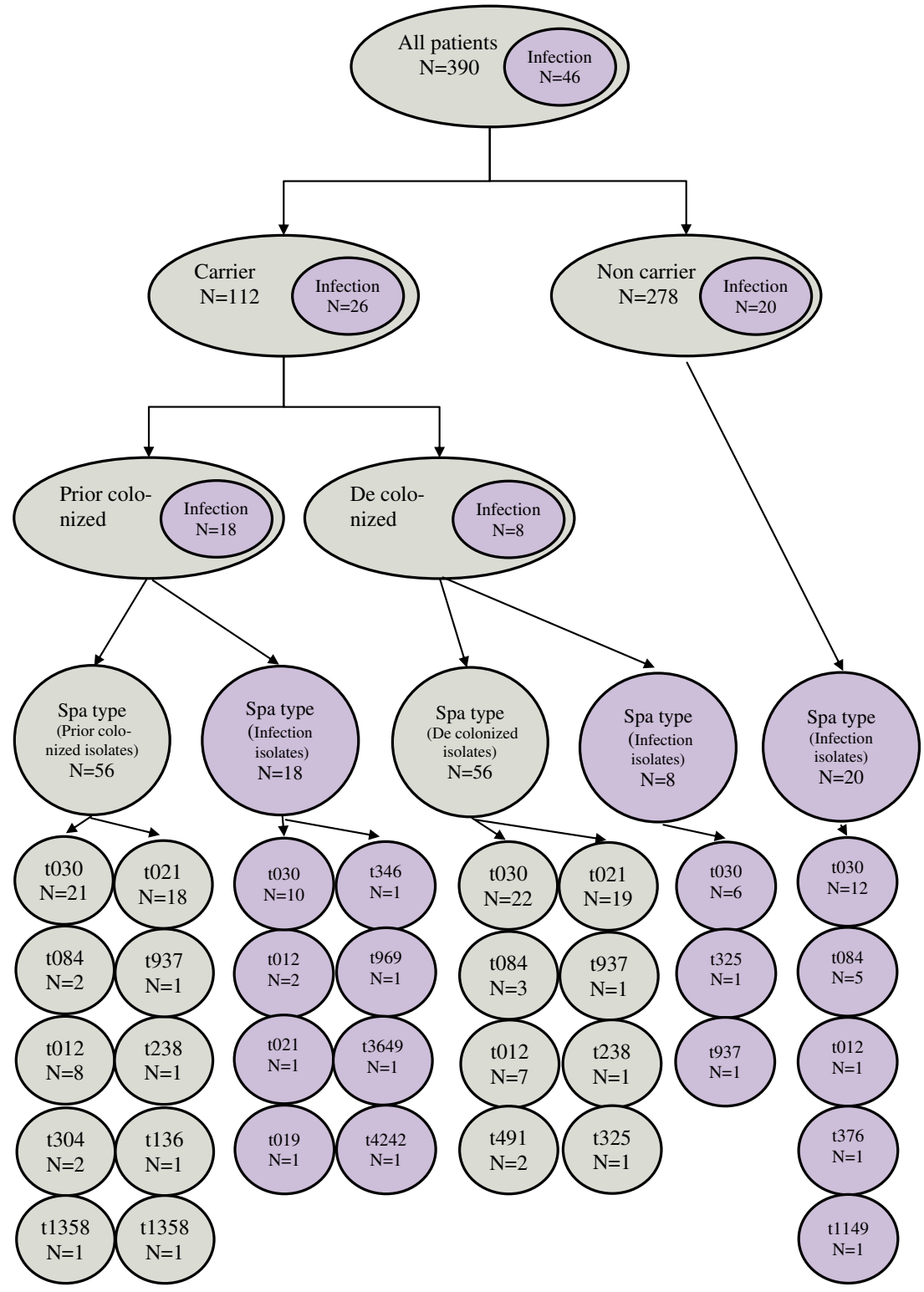

\section{Concluding statement}

This study again demonstrates that $S$. aureus nasal colonization is significantly associated with infection. Although many studies showed that $S$. aureus infection is of an endogenous nature, our results indicate that the source of ICU S. aureus infection can as well be exogenous and a dominant and locally circulating MRSA clone plays an important role in our ICU infections. Given the apparent fact that $S$. aureus causes serious problems in the hospital setting, the use of appropriate measures to prevent the transmission of these strains between patients, personnel, and hospital environment is necessary. We show that nasal $S$. aureus decolonization during ICU stay protects patients against infection [6], even in the presence of easily circulating nosocomial MRSA clones.
Acknowledgments This work was financially supported by the Arak University of Medical Sciences, Iran, for which we are thankful. This paper is extracted from a dissertation by Leila Akhtar Danesh to fulfill the requirement for Master of Sciences in Medical Bacteriology and Zeinab Saiedi Nejad to become a specialist in infectious diseases.

Authors' contribution EGR, AVB, and HS conceptualized, designed, and supervised the study. LA and SF were involved in the sample collection and performed laboratory experiments. ZS managed the decolonization of patients and all other activities in the ICUs. All the authors contributed in writing and editing of the draft and have seen and agreed to the submitted version of the paper.

\section{Compliance with ethical standards}

Conflict of interest AVB is an employee of BioMérieux, a company designing, developing, and selling infectious disease tests. The authors report no other conflicts of interest. 
Disclaimer The company had no influence on the design and execution of the study or in the writing of the manuscript.

\section{References}

1. Van Rijen MM, Bode LG, Baak DA, Kluytmans JA, Vos MC (2012) Reduced costs for Staphylococcus aureus carriers treated prophylactically with mupirocin and chlorhexidine in cardiothoracic and orthopaedic surgery. Plos ONE 7(8):e43065

2. Bakthavatchalam YD, Nabarro LEB, Ralph R, Veeraraghavan B (2017) Diagnosis and management of Panton-Valentine leukocidin toxin associated Staphylococcus aureus infection: an update. Virulence 0 https://doi.org/10.1080/21505594.2017.1362532

3. Stapleton PD, Taylor PW (2002) Methicillin resistance in Staphylococcus aureus: mechanisms and modulation. Sci Prog 85(1):57-72

4. Lamaro-Cardoso J, De Lencastre H, Kipnis A et al (2009) Molecular epidemiology and risk factors for nasal carriage of Staphylococcus aureus and methicillin-resistant $S$. aureus in infants attending day care centers in Brazil. J Clin Microbiol 47(12):3991-3997

5. Ghasemzadeh-Moghaddam H, Neela V, van Wamel W et al (2015) Nasal carriers are more likely to acquire exogenous Staphylococcus aureus strains than non-carriers. Clin Microbiol Infect 21(11):998. e991-998. e997

6. Bode LG, Kluytmans JA, Wertheim HF, Bogaers D, Vandenbroucke-Grauls CM, Roosendaal R, Troelstra A, Box AT, Voss A, van der Tweel I, van Belkum A, Verbrugh HA, Vos MC (2010) Preventing surgical-site infections in nasal carriers of Staphylococcus aureus. N Engl J Med. 362(1):9-17

7. Asadollahi P, Farahani NN, Mirzaii M, Khoramrooz SS, van Belkum A, Asadollahi K, Dadashi M, Darban-Sarokhalil D (2018) Distribution of the most prevalent spa types among clinical isolates of methicillin-resistant and -susceptible Staphylococcus aureus around the world: a review. Front Microbiol. 9:163

8. Scully BE, Briones F (1992) Gu J-w, Neu HC. Mupirocin treatment of nasal staphylococcal colonization. Arch Intern Med 152(2):353-356

9. Fooladvand S, Sarmadian H, Habibi D, van Belkum A, GhaznaviRad E (2019) High prevalence of methicillin resistant and enterotoxin gene-positive Staphylococcus aureus among nasally colonized food handlers in central Iran. Eur J Clin Microbiol Infect Dis 38(1):87-92

10. Fard-Mousavi N, Mosayebi G, Amouzandeh-Nobaveh A, JapouniNejad A, Ghaznavi-Rad E (2015) The dynamic of Staphylococcus aureus nasal carriage in Central Iran. Jundishapur J Microbiol 8(7): e20760

11. Na'Was T, Hawwari A, Hendrix E et al (1998) Phenotypic and genotypic characterization of nosocomial Staphylococcus aureus isolates from trauma patients. J Clin Microbiol 36(2):414-420

12. Ho P-L (2003) Carriage of methicillin-resistant Staphylococcus aureus, ceftazidime-resistant Gram-negative bacilli, and vancomycin-resistant enterococci before and after intensive care unit admission. Crit Care Med 31(4):1175-1182

13. Cabalak M, Kalkan A, Inci NA, Caliskan A, Demirdag K (2013) Clonal relation of nasal MRSA carrier status among hospital personnel, hospitalized patients and community. J Microbiol Infect Dis 3(2):49-55

14. Korn GP, Martino MD, Mimica IM, Mimica LJ, Chiavone PA, LRdS M (2001) High frequency of colonization and absence of identifiable risk factors for methicillin-resistant Staphylococcus aureus (MRSA) in intensive care units in Brazil. Brazilian J Infect Dis 5(1):1-7

15. Maleki Z, Anjerani S (2006) Comparison of the results of antimicrobial susceptibility with the disk diffusion and $\mathrm{E}$ test method for oxacillin and vancomycin antibiotics. Islamic Azad Uni J Med Sci 16(4):211-215
16. Afrough P, Pourmand MR, Zeinalinia N, Yousefi M, Abdossamadi Z, Bagherzadeh YS (2012) Molecular typing of clinical and nasal carriage isolates of Staphylococcus aureus by spa gene patterns. J Mazandaran Uni Med Sci 22(94):28-34

17. Garrouste-Orgeas M, Timsit J-F, Kallel H et al (2001) Colonization with methicillin-resistant Staphylococcus aureus in ICU patients morbidity, mortality, and glycopeptide use. Infect Control Hosp Epidemiol 22(11):687-692

18. Corbella X, Domíguez M, Pujol M et al (1997) Staphylococcus aureus nasal carriage as a marker for subsequent staphylococcal infections in intensive care unit patients. Eur J Clin Microbiol Infect Dis 16(5):351-357

19. Ayepola OO, Olasupo NA, Egwari LO, Becker K, Schaumburg F (2015) Molecular characterization and antimicrobial susceptibility of Staphylococcus aureus isolates from clinical infection and asymptomatic carriers in Southwest Nigeria. Plos ONE 10(9):e0137531

20. Khosravi AD, Hoveizavi H, Farshadzadeh Z (2012) The prevalence of genes encoding leukocidins in Staphylococcus aureus strains resistant and sensitive to methicillin isolated from burn patients in Taleghani Hospital, Ahvaz Iran. Burns. 38(2):247-251

21. Nashev D, Toshkova K, Bizeva L, Akineden Ö, Lämmler C, Zschöck M (2007) Distribution of enterotoxin genes among carriage-and infection-associated isolates of Staphylococcus aureus. Letters Appl Microbiol 45(6):681-685

22. Gadyari F, Sattari M, Boroumand MA, Yaghoubi R, Sepehriseresht S, Purgholi L (2011) Detection of Staphylococcus aureus enterotoxins A to D in clinical strains isolated from burned patients of Tehran Motahari Hospital. Iranian J Med Microbiol 5(1):20-27

23. Normanno G, La Salandra G, Dambrosio A et al (2007) Occurrence, characterization and antimicrobial resistance of enterotoxigenic Staphylococcus aureus isolated from meat and dairy products. Int J Food Microbiol 115(3):290-296

24. Ghasemian A, Peerayeh SN, Bakhshi B, Mirzaee M (2015) The microbial surface components recognizing adhesive matrix molecules (MSCRAMMs) genes among clinical isolates of Staphylococcus aureus from hospitalized children. Iranian J Pathol 10(4):258

25. Duran N, Dogramaci Y, Ozer B, Demir C, Kalaci A (2010) Detection of adhesin genes and slime production among staphylococci in orthopaedic surgical wounds. Afr J Microbiol Res 4(9):708-715

26. Mirzaii M, Emaneini M, Jabalameli F, Halimi S, Taherikalani M (2015) Molecular investigation of Staphylococcus aureus isolated from the patients, personnel, air and environment of an ICU in a hospital in Tehran. J Infect Pub Health 8(2):202-206

27. Goudarzi M, Seyedjavadi SS, Azad M, Goudarzi H, Azimi H (2016) Distribution of spa types, integrons and associated gene cassettes in Staphylococcus aureus strains isolated from intensive care units of hospitals in Tehran, Iran. Arch Clin Infect Dis 11(4):e38813

28. Chen H, Liu Y, Jiang X, Chen M, Wang H (2010) Rapid change of methicillin-resistant Staphylococcus aureus clones in a Chinese tertiary care hospital over a 15 -year period. Antimicrob Agent Chemother 54(5):1842-1847

29. Goudarzi M, Fazeli M, Goudarzi H, Azad M, Seyedjavadi SS (2016) Spa typing of Staphylococcus aureus strains isolated from clinical specimens of patients with nosocomial infections in Tehran, Iran. Jundishapur J Microbiol 9(7):e35685

30. Havaei SA, Ghanbari F, Rastegari AA, et al (2014) Molecular typing of hospital-acquired Staphylococcus aureus isolated from Isfahan, Iran. Int Schol Res Notices 2014:6. https://doi.org/10.1155/2014/185272

31. Japoni A, Jamalidoust M, Farshad S et al (2011) Characterization of SCCmec types and antibacterial susceptibility patterns of methicillin-resistant Staphylococcus aureus in Southern Iran. Jap J Infect Dis 64(1):28-33

Publisher's note Springer Nature remains neutral with regard to jurisdictional claims in published maps and institutional affiliations. 